\title{
Una experiencia de comunidad de aprendizaje virtual. El uso de nuevas TIC en el desarrollo colaborativo de recursos WWW para una asignatura*
}

\author{
Francisco J. Miguel Quesada \\ Universitat Autònoma de Barcelona \\ miguel.quesada@uab.cat
}

Recibido: 12-12-2008

Aceptado: 15-05-2008

\section{Resumen}

Se presenta una experiencia de innovación docente dirigida a potenciar las competencias de búsqueda, evaluación crítica y gestión de la información, junto con otras estrategias cooperativas de aprendizaje y resolución de problemas. Se muestra el uso de diversas TIC en la elaboración cooperativa de un glosario de términos fundamentales para una asignatura. Se constata la generación de una dinámica de colaboración entre el alumnado de una asignatura en cuanto a las tareas de lectura, selección, propuesta y definición de términos. Como resultado, al mismo tiempo que refuerza competencias instrumentales básicas respecto al nivel exigido por el plan docente en cuanto a TICs, el alumnado realiza de forma efectiva un proceso de aprendizaje cooperativo mientras se implica en la construcción y la difusión de un recurso de utilidad pública (el glosario completo).

Palabras clave: aprendizaje cooperativo, comunidades virtuales de aprendizaje, uso didáctico de las nuevas TICs, entornos bimodales de aprendizaje.

Abstract. A virtual learning community experience: The use of new information and communication technologies for collaborative developing WWW resources in learning contexts

The present research note presents an educational innovation experience that aims to enforce the competences on searching, evaluating and managing relevant information, combined with other strategies for cooperative learning and problem-solving. A number of ICTs will be used in the cooperative development of a glossary of key terms for a course subject. It will be showed how this generates an emergent pattern of dynamic collaboration among students, related to the tasks of reading, selecting, and proposing definitions of key terms. As a result the students, putting in practice basic skills according to the ICT level required by the subject, are involved in a process of cooperative learning while they are committed in the construction and dissemination of a resource of public utility (the full glossary).

Key words: cooperative learning, virtual communities of learning, use of new ICT technologies, bi-modal learning environments.

* El presente trabajo se ha publicado en el marco de un proyecto I+D financiado por el Ministerio de Educación y Ciencia y el FEDER, con referencia SEJ2006-00959/SOCI. 


\author{
Sumario \\ 1. Objetivos 3. Conclusiones: Revisión \\ 2. Descripción de la experiencia \\ de la experiencia \\ Bibliografia
}

\title{
1. Objetivos
}

El objetivo inicial de la experiencia didáctica aquí presentada es abordar un conjunto de problemas detectados durante el desarrollo de la práctica docente en la Facultad de Ciencias Políticas i Sociología de la Universidad Autónoma de Barcelona. Durante años, esta facultad ha intentado responder a la problemática específica que afecta al numeroso alumnado matriculado en el grupo 51, esto es, estudiantes con sesiones presenciales por la tarde. La mayoría de estos responden al perfil de quien compatibiliza sus estudios con un trabajo no esporádico.

Una parte de la problemática general atañe al cambio de orientación desde la enseñanza hacia el aprendizaje. Con este cambio de perspectiva, el alumnado pasa de ser un receptor pasivo a ser un agente investigador con un papel muy activo en su propio proceso de aprendizaje, mientras que el profesorado pasa de ser una fuente de sabiduría a ser un activo guía de tales procesos de aprendizaje. Las nuevas formas de entender el uso docente de las llamadas nuevas tecnologias de la información y la comunicación (NTIC), que, implementadas dentro de un grupo social, se constituyen en «nuevos ambientes de aprendizaje», van en la línea de implantar efectivamente una cultura colaborativa en las instituciones educativas. Tal perspectiva es promovida activamente en la mayoría de instituciones de educación superior e implica incidir especialmente sobre las condiciones en que se encuentra el alumnado, con la finalidad de proporcionarle un entorno adecuado en el que pueda desarrollar su propio camino hacia la formación, abandonando la idea de mera transmisión de conocimientos según el modelo de clase magistral hegemónica (Harasim et al., 2002: 198).

Conviene, sin embargo, destacar otra parte de la problemática: la especificidad propia de los grupos de tarde. Este alumnado se enfrenta a una situación especial en el aula respecto a la cuestión de su motivación. Dado que, en su mayoría, acceden a las sesiones tras una jornada laboral más o menos intensa, sus capacidades de atención y rendimiento están en ocasiones considerablemente limitadas. También cabe considerar como problemática específica el mero hecho de la falta de presencialidad del alumnado, que participa en las sesiones con un alto grado de dispersión en cuanto a su asistencia. Todo esto supone la necesidad de ajustar las prácticas docentes a un escenario formativo en educación superior en el que la presencialidad no es el contexto predominante y debe ser combinada con otras modalidades formativas.

En tercer lugar, cabe considerar una serie de problemas «nuevos» en el contexto universitario y social actual. La llamada «sociedad de la información y la 
comunicación» marca unas nuevas necesidades en relación con las capacidades requeridas para el nivel formativo superior (Kozma y Schank, 2000). Estas nuevas capacidades y habilidades tienen dos vertientes fundamentales, una instrumental, o tecnológica, y otra relativa a hábitos de organización del trabajo. La institución universitaria debe enfrentarse al reto de proporcionar un nivel de competencias instrumentales mínimas, pero esta exigencia no puede verse tan sólo como un impedimento o como un problema añadido actualmente a los «viejos» problemas de la docencia. Precisamente, la nueva situación puede percibirse como una ocasión y un beneficio para las prácticas docentes (Cabero, 1998). Así, las nuevas herramientas tecnológicas suponen un potencial de innovación en el aula, siempre que se vinculen estrechamente a los contenidos sustantivos, esto es, siempre que se puedan desarrollar modelos de integración curricular en las asignaturas. Las instituciones universitarias no deben agotar su esfuerzo en su propia actualización tecnológica o en proporcionar unas habilidades instrumentales a sus usuarios finales, sino que deben integrar estas nuevas tecnologías, y el nuevo contexto tecnológico y social (e.g., la no-presencialidad) dentro de sus contenidos propios, como una herramienta más dentro de su tarea docente. Como indica Cabero, cualquier TIC «[...] es simplemente un instrumento curricular más, de manera que su posible eficacia no va a depender exclusivamente de su potencialidad tecnológica para transmitir, manipular e interaccionar información, sino también, y puede que sea lo significativo, del curriculum en el cual se introduzca, de las relaciones que establezca con otros elementos curriculares, y de otras medidas, como el papel que desempeñen el profesor y el alumno en el proceso formativo» (1998: 1145).

En este último sentido, muchas universidades están trabajando sobre un modelo específico de enseñanza superior que aborde los problemas expresados. Este modelo es el que en nuestra universidad se ha dado en denominar «bimodal» (Yábar y Barbará, 1999). Se trata de una combinación de las posibilidades que ofrecen las nuevas TIC con las prácticas docentes tradicionales en el aula (clases magistrales, prácticas, etc.). Su objetivo genérico es conseguir un mejor ajuste de la formación superior a las necesidades del colectivo al que va dirigida, y también al contenido sustantivo de cada una de las disciplinas implicadas.

La experiencia de innovación docente que aquí se está presentando se inscribe dentro del conjunto de propuestas que responden a las especificaciones de este modelo «bimodal» de aprendizaje, pero va más allá del mismo, al considerar que la presencialidad (o la no-presencialidad) no es un dato contextual, o una mera posibilidad tecnológica, sino que, en determinados contextos (como es el caso para el alumnado de tarde), es una necesidad marcada por esta nueva estructura sociolaboral de las sociedades de la información y la comunicación. Son los problemas reales de este alumnado para asistir a las sesiones en el aula y los requerimientos de desarrollo de nuevas habilidades lo que debe orientar el acto formativo dirigido a tal colectivo.

El objetivo específico perseguido por la experiencia implica que el alumnado adquiera conocimientos pertinentes de cada asignatura concreta, y que lo 
haga de forma integrada con otras habilidades de carácter instrumental vinculadas tanto al uso de las nuevas TIC, como al desarrollo de hábitos de trabajo colaborativos y de carácter «virtual» o telemático. Se ejemplificará a continuación un modo en el que las competencias requeridas en la sociedad de la información y la comunicación pueden incorporarse en las asignaturas, mediante la programación de prácticas integradas que promueven un aprendizaje a partir de un trabajo práctico que: 1) se basa en la cooperación y 2) promueve la cooperación. El trabajo individual y colectivo no presencial, programado con objetivos didácticamente relevantes, supone un marco especialmente interesante para experimentar con formatos docentes semipresenciales gracias a las posibilidades de interacción que proporcionan las nuevas TIC. Pero la experiencia no se restringe a una mera aplicación de herramientas tecnológicas, sino que también pretende incidir especialmente en los aspectos, esencialmente sociales, de: 1) el desarrollo de capacidades de organización conjunta del trabajo, 2) la motivación del proceso de aprendizaje mediante la participación activa en la creación de recursos útiles dotados de persistencia más allá del curso y 3) la promoción de un cierto espiritu de grupo dentro del alumnado, que no necesariamente precisa de una copresencia en el aula, sino que se acerca más bien al modelo de "comunidad virtual de aprendizaje» que la existencia de WWW permite (y, tal vez, exige, de cara al futuro) ${ }^{1}$.

En el diseño de la experiencia docente, se tuvo muy en cuenta que las cuestiones fundamentales respecto a la formación se han desplazado, desde la transmisión de conocimientos clásica, hacia un nuevo contexto marcado por: 1) la libre disponibilidad de la información y 2) la eficaz gestión de la misma. Por ello, el valor añadido de una formación universitaria especializada debería incidir destacadamente, en el momento actual, en desarrollar de forma efectiva la adquisición de competencias relativas a las estrategias de búsqueda de la información, a su evaluación crítica y a las formas de procesamiento de la misma. El objetivo final es el declarado por Salmon $(1998,2000)$ en su modelo de cinco etapas hacia el «aprendizaje independiente»: 1) acceso y motivación (el profesor dará instrucciones sobre cómo usar el sistema e intentará construir la confianza de los usuarios, animándolos a que sigan hacia delante), 2) socialización en línea (se debe perseguir la cohesión del grupo), 3) intercambio de información (se animará a todos los participantes a contribuir en la discusión, y a «tejer» la información), 4) construcción del conocimiento (se promoverá la construcción de conocimiento, promoviendo posiciones que permitan aprender de los problemas encontrados) y 5) desarrollo personal (se pretende que el estudiante alcance autonomía para continuar con su proceso de aprendizaje). Este modelo, referido originariamente al aprendizaje moderado electrónicamente, se ha tenido muy en cuenta a la hora de diseñar la presente experiencia docente.

1. Sobre comunidades virtuales de aprendizaje, véase Palloff y Pratt (1999). 


\section{Descripción de la experiencia}

La experiencia se centra alrededor de la elaboración cooperativa de un glosario de términos fundamentales para una asignatura concreta. El caso presentado es el de la asignatura Metodología de las Ciencias Sociales (de carácter obligatorio en el primer cuatrimestre del segundo curso de la licenciatura de Sociología). En la Facultad de Ciencias Políticas i Sociología de la UAB, durante el curso de referencia (2003-2004), la asignatura se impartió en grupos de alumnos que acuden en horario de mañana o por la tarde. Este último grupo es el seleccionado para llevar a cabo la primera fase de la experiencia planteada.

La planificación docente implica dos fases temporales diferenciadas a lo largo de las cuales tienen lugar diversos procesos, algunos de los cuales son simultáneos, según muestra el esquema de la tabla 1 .

La fase previa consiste en la preparación de los materiales para el curso, esto es, la selección de la bibliografía y de las direcciones de los recursos WWW pertinentes, así como la recopilación y la edición de un conjunto de lecturas cortas organizadas temáticamente ${ }^{2}$. Estos materiales son los que se usan para la

Tabla 1. Fases del proceso de construcción de un glosario

\begin{tabular}{|c|c|c|c|}
\hline Fases & Docente & Alumnado & Resultado \\
\hline Previa & $\begin{array}{l}\text { (1) Preparación } \\
\text { de materiales. }\end{array}$ & & $\begin{array}{l}\text { - Dossier de lecturas. } \\
\text { - Bibliografía. } \\
\text { - Recursos WWW. }\end{array}$ \\
\hline \multirow{3}{*}{ Primera } & & $\begin{array}{l}\text { (2) Revisión de } \\
\text { los materiales. }\end{array}$ & \multirow{3}{*}{$\begin{array}{l}\text { Listado de términos } \\
\text { para el glosario. }\end{array}$} \\
\hline & $\begin{array}{l}\text { (4) Revisión del } \\
\text { listado. }\end{array}$ & $\begin{array}{l}\text { (3) Propuesta de } \\
\text { términos vía } \\
\text { correo electrónico. }\end{array}$ & \\
\hline & (5) Edición en web. & & \\
\hline \multirow{3}{*}{ Segunda } & & $\begin{array}{l}\text { (6) Búsqueda de } \\
\text { definiciones y citas. }\end{array}$ & \multirow{3}{*}{ Glosario de términos. } \\
\hline & $\begin{array}{l}\text { (8) Revisión de } \\
\text { definiciones } \\
\text { y citas. }\end{array}$ & $\begin{array}{l}\text { (7) Envío vía } \\
\text { correo electrónico. }\end{array}$ & \\
\hline & (9) Edición en web. & & \\
\hline
\end{tabular}

2. Estas lecturas se usan para otra práctica docente integrada: los seminarios de discusión, en los que varios alumnos voluntarios asumen la defensa de las posturas contrapuestas que expresan las lecturas, en una discusión pública argumentada ante el resto de la clase. 
totalidad del curso y, por tanto, no presentan ninguna especificidad en relación con la práctica docente aquí presentada.

\subsection{Primera fase: la construcción del listado de términos para el glosario}

En la primera fase, planteada al inicio del curso y desarrollada hasta inicios de diciembre, los participantes y las participantes deben revisar los materiales propios de la asignatura (disponibles en los dossiers de fotocopias, en la documentación en línea o en las referencias de la bibliografía del curso), con el objetivo de elaborar listas de términos que estimen relevantes para el conjunto de la materia. La inclusión de un término en el glosario supone su selección por parte del alumnado en función de dos criterios: la relevancia estimada o bien el actual desconocimiento del término ${ }^{3}$.

Esta primera fase se cierra cuando todas las listas son enviadas por correo electrónico al responsable docente, dentro de un plazo temporal límite. Cada estudiante que desea participar en la experiencia envía sus propias propuestas, y corresponde al responsable docente construir y actualizar periódicamente (de forma ideal, diariamente) una página web con el listado de los términos propuestos hasta el momento. El alumnado participante puede consultar qué términos han sido ya propuestos para ajustar su lista y, de este modo, proponer sólo términos «nuevos».

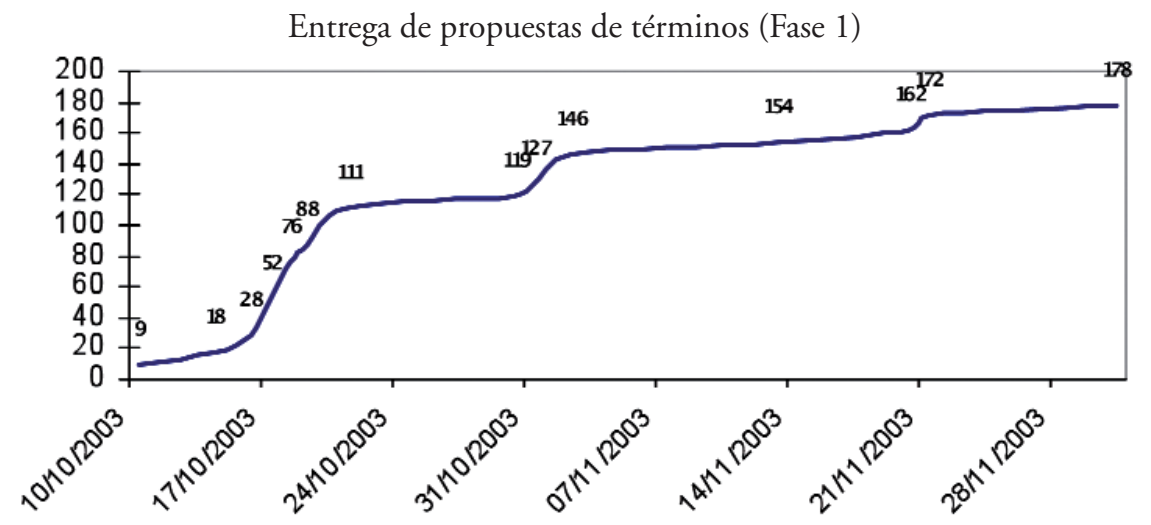

Ilustración 1. Participación en la fase 1 de la experiencia docente.

3. Se recomienda como criterio que se incluyan en la lista aquellos términos que cada uno desearía tener definidos en el momento de enfrentarse al examen final del curso. 


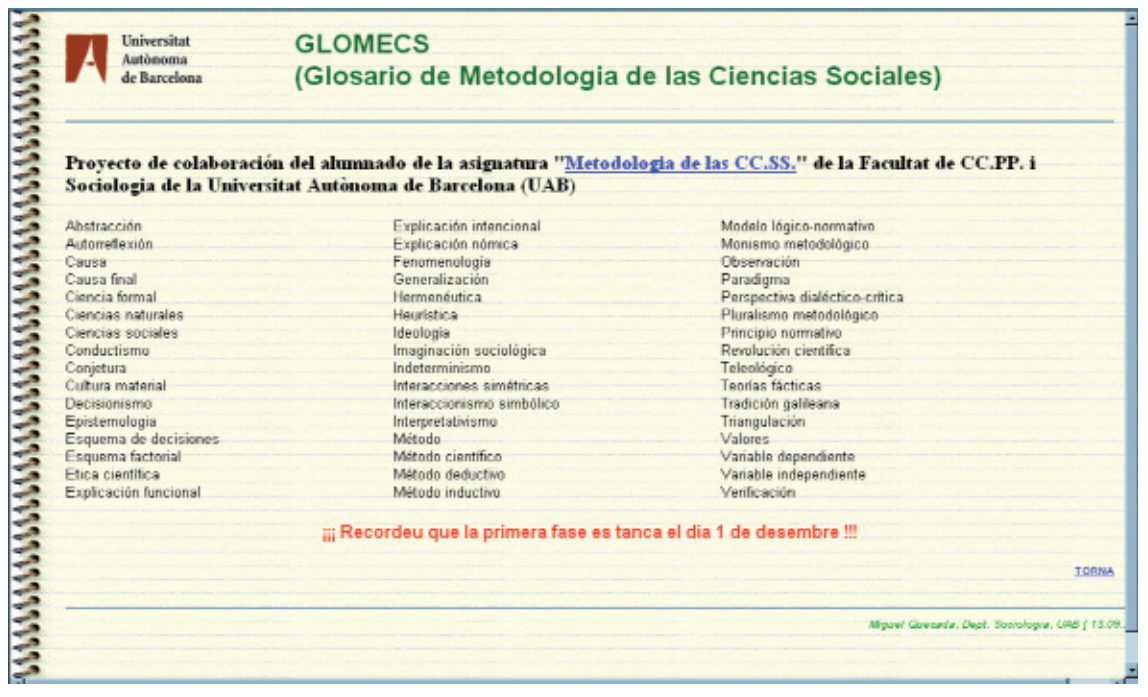

Ilustración 2. Ejemplo de listado parcial de términos, consultable vía web por los alumnos.

Se puede constatar como, en poco tiempo, se crea una dinámica de participación en el trabajo cooperativo (con envíos escalonados) o de confección del listado de términos, bien sea virtual, mediante consulta a los listados provisionales, bien sea real, mediante la coordinación y la constitución de grupos informales autoorganizados de revisión de los materiales. El alumnado participante optimiza la revisión bibliográfica, mientras se asegura que la lista propuesta sea aceptada íntegramente. Se piden diez términos por alumno y se estimula el envío escalonado para evitar la «devolución de términos» (que se produce cuando un alumno propone un término ya propuesto o en el caso extremo en que el profesor deba rechazar un término por irrelevante).

Tras ocho semanas de revisión de materiales y propuesta de términos, se cierra el plazo de entrega y se evalúa la participación del alumnado a partir de los registros sobre las fechas de entrega y la integridad de las listas aceptadas. Desde el inicio del curso, se estipuló que la participación en esta actividad era totalmente voluntaria y que sumaría hasta un total de dos puntos a la nota final de la asignatura. La participación en esta primera experiencia fue del $46,3 \%$ del alumnado matriculado, lo que parece indicar un nivel bajo de aceptación de la propuesta, pero resulta más interesante analizar los datos en relación con el nivel de asistencia a las clases. Referente al alumnado matriculado (41), hay una parte que no asiste a las clases $(51,2 \%)$ ni se presenta al examen final $(58,5 \%)$. Si se descuenta este subgrupo, las proporciones de participación en las actividades voluntarias resultan elevadas. Así, un 70\% de los asistentes a clase participaron en los seminarios de discusión. En relación con 
la experiencia aquí presentada, un $95 \%$ de los asistentes propuso términos y un $70 \%$ culminó la práctica (segunda fase). Cabe destacar que casi un tercio de los asistentes a las clases lo fueron de forma irregular a lo largo de todo el curso, sin embargo, su participación en las prácticas integradas les proporcionó una forma de asistencia continuada, así como un ámbito de relación con sus compañeros y compañeras, y contribuyó a su evaluación final de la asignatura.

Hay que destacar que la tarea del responsable docente durante esta fase es fundamental y continua ${ }^{4}$. En caso de utilizar una herramienta de creación de contenido sin intervención por parte del alumnado, se debe mantener la página web correspondiente al listado de términos actualizada casi diariamente, para que sea posible comprobar los términos que han sido propuestos hasta el momento. En cualquier caso, resulta esencial establecer de forma clara una página WWW como el «espacio de referencia» del proyecto, esto es, como un «aula virtual» en la que se encuentren los diversos alumnos y el docente colaborando en el proyecto común. Finalmente, el papel del profesor, como experto en la materia sustantiva del curso, resulta fundamental en esta fase para que los términos realmente relevantes de la asignatura se encuentren entre los seleccionados, o bien añadirlos por su cuenta al listado si no han aparecido tras la revisión del material realizada por el alumnado.

\subsection{Segunda fase: la definición de los términos del glosario}

En la segunda fase, que se extiende hasta el final del curso, el responsable docente reparte entre los participantes el conjunto de términos seleccionados, de forma que nadie reciba un término que ese mismo alumno/a propusiera anteriormente. El objetivo es que cada participante se responsabilice de la definición de diez términos, que se constituirán en otras tantas entradas del glosario final. El trabajo incluye tres apartados requeridos para la evaluación de cada término del glosario: 1) una definición breve del término, redactada por el alumno o la alumna con sus propias palabras (se previene contra el uso de lenguaje excesivamente técnico y contra la copia directa desde las fuentes); 2) una cita textual en que se haga uso del término (en el sentido de la definición propuesta), y 3) la referencia completa de la autoría de la cita.

A medida que se envían las definiciones, el responsable docente las evalúa y las acepta como definitivas si se cumplen los criterios de adecuación para ser publicadas como glosario definitivo. El resto son «devueltas» a los participantes para que continúen con su trabajo hasta completarlas. Todo este proceso de comunicación se llevó a cabo mediante el uso del correo electrónico.

4. Sobre las habilidades del profesor en estos nuevos entornos, puede consultarse Marqués (1999: 97). 
Definiciones del glosario completadas (Fase 2)

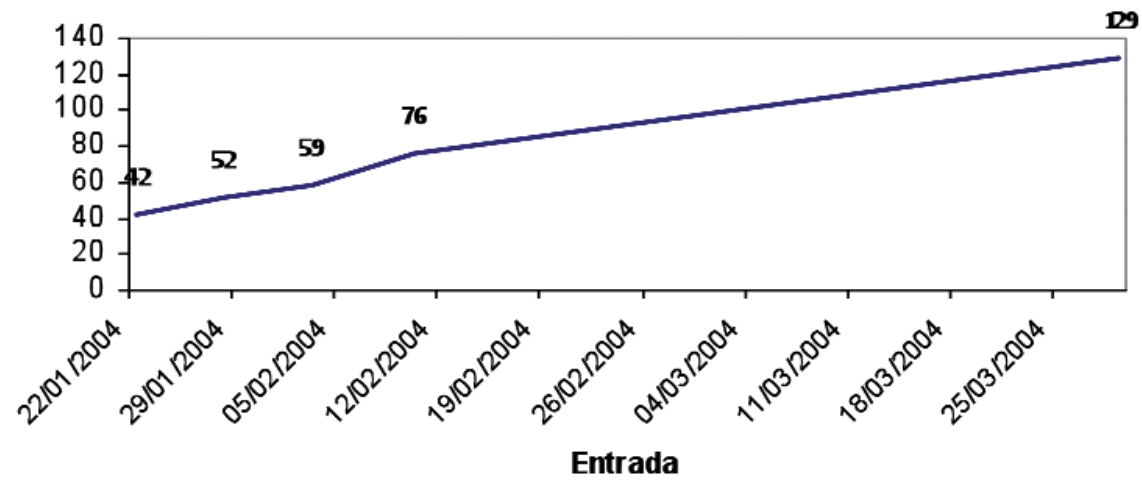

Ilustración 3. Participación en la fase 2 de la experiencia docente.

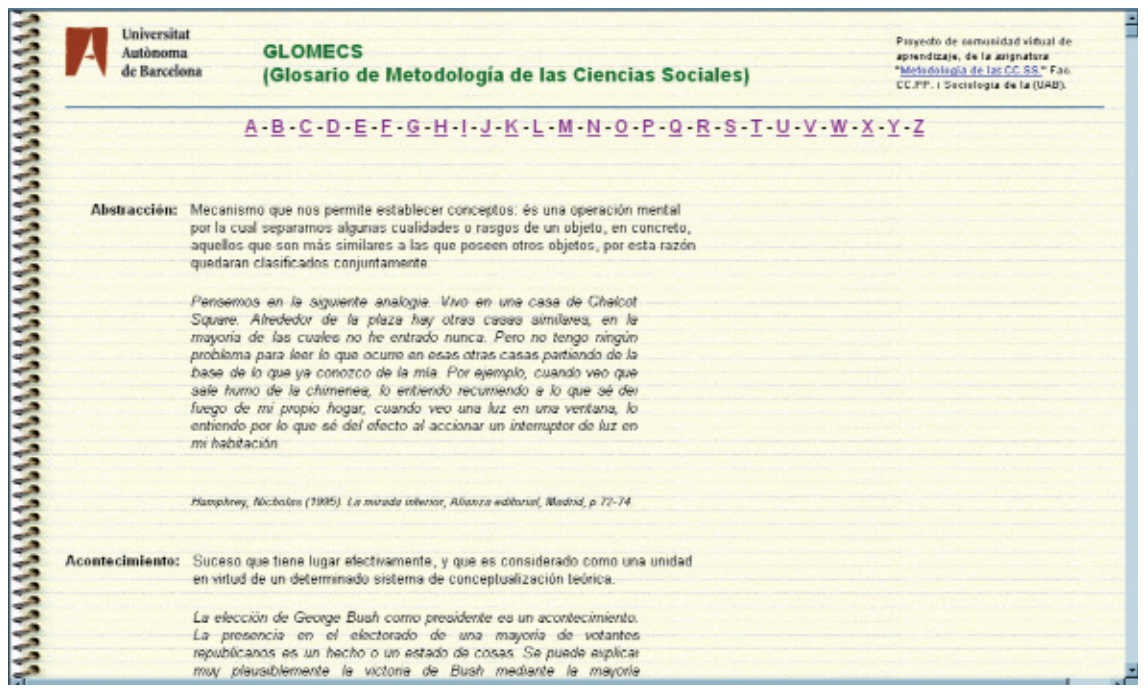

Ilustración 4. Ejemplo de listado final de términos, consultable vía web.

El proceso correspondiente a esta experiencia docente culmina con la publicación electrónica del glosario completo durante la semana previa a los exámenes de la asignatura. El conjunto del alumnado puede consultar este recurso para la preparación de las pruebas de evaluación escrita, que incluso podría quedar disponible como parte de la «base de conocimientos» del departamento correspondiente. 


\section{Conclusiones: Revisión de la experiencia}

A lo largo de todo este proceso, se ha generado un conjunto de evidencias del proceso de aprendizaje y el responsable docente puede evaluar la participación individual de cada estudiante, ya que tiene constancia de su participación en el proyecto mediante indicadores tales como las fechas de entrega, la cantidad de términos «relevantes» aportados y la calidad de sus definiciones y citas. Supone, por tanto, un excelente complemento a la evaluación general de la asignatura ${ }^{5}$. Por su parte, el alumnado, mediante la participación en esta práctica integrada dentro del contenido curricular de la asignatura: 1) refuerza sus vínculos, como comunidad de aprendizaje, con el resto de compañeros y compañeras; 2) desarrolla sus competencias de búsqueda y comunicación telemática, y 3) desarrolla su conocimiento de los materiales fundamentales, del vocabulario y de la estructura básica de la asignatura. A lo largo de un periodo de cuatro meses, cada participante lleva a cabo una tarea concreta que combina actividades presenciales y virtuales en un entorno de trabajo cooperativo «bimodal». Las actividades presenciales en el aula implican la coordinación de todo el proyecto, así como sesiones de discusión pública sobre la relevancia de algunos términos propuestos (que el responsable docente promueve en sesiones dedicadas). Las actividades virtuales implican la búsqueda de información, la comunicación y el trabajo cooperativo a través de recursos NTIC.

La primera experiencia piloto, realizada durante el curso 2003-2004, mostró cómo tal programación promueve efectivamente un conjunto de competencias relativas al trabajo colaborativo, así como la creación de un nuevo espacio de interacción social mediada por ordenadores (Ryan et al., 2000) que amplía las posibilidades docentes del aula, especialmente en contextos de semipresencialidad, como el del grupo de tarde aquí considerado. Igualmente, la experiencia mostró un modelo de integración del uso de nuevas TIC dentro de los contenidos concretos de una asignatura, de modo que permite insistir sobre la capacitación ${ }^{6}$ en cuanto a: 1) búsqueda de información, 2) evaluación crítica de la misma y 3) usos tecnológicos y de gestión del conocimiento.

Cabe destacar, por otro lado, que esta estrategia requiere una importante labor de coordinación entre asignaturas, que puede articularse a través de la incorporación al proyecto más amplio de Acreditación $\mathrm{ECDL}^{7}$. La decisión

5. La evaluación se realiza a través de una prueba objetiva de diez preguntas cortas, más el desarrollo extenso de un tema a elegir entre cuatro propuestas vinculadas temáticamente a los seminarios de discusión.

6. Fundamental entre el alumnado de un primer ciclo universitario. Estos puntos forman parte integral del currículo del plan docente de la facultad de referencia, en línea con la evitación de oferta de asignaturas o complementos específicamente dedicados a la formación tecnológica. Por el contrario, se promueven formas innovadoras de integrar la formación en nuevas TIC dentro de asignaturas concretas y sustantivas, según el modelo de aprendizaje mediante la resolución práctica de proyectos concretos (Roblyer et al., 1997).

7. Para más información sobre la acreditación European Computer Driver Licence (ECDL), puede consultarse http://www.ecdl.com (30/03/2004) o http://ecdl.ati.es (30/03/2004). 
de qué herramientas tecnológicas recibirán atención dentro del plan docente concreto de cada licenciatura universitaria viene marcada por los protocolos internacionales al respecto, pero la programación del modo de llevar a cabo la formación y las prácticas al respecto depende de una coordinación que sólo se puede llevar a cabo a nivel de facultad.

El principal aspecto destacable, de carácter didáctico, es la efectividad de esta experiencia para generar estrategias cooperativas de aprendizaje y resolución de problemas entre el alumnado. Tal efectividad se detectó como efecto no esperado en el diseño de la primera experiencia docente $(2003-2004)^{8}$ y se ha podido confirmar en la segunda aplicación (2008-2009). A la vista del resultado, en la primera experiencia piloto se percibió una tendencia entre el alumnado participante: 1) a formar grupos para la búsqueda de términos, definiciones y citas, con un considerable flujo de información cruzada entre ellos, y 2) a participar en mayor medida de la esperada en actividades opcionales del curso. Así, se podría entender como «subproducto» del proyecto original sobre introducción de NTIC en la asignatura, la generación de una dinámica intensa de colaboración por parte de los participantes y las participantes en cuanto a las tareas de lectura, selección, propuesta y definición de términos. Así mismo, la participación parece reforzada por la motivación adicional de sentirse implicados en la construcción y la difusión de un recurso de utilidad pública (esto es, el glosario completo). Si tales efectos, en general, son deseables para el conjunto del alumnado, lo son tanto más para los grupos de tarde, que cuentan con las especificidades citadas al inicio de la presente comunicación.

A partir de esta prueba piloto, se establecieron tres líneas de continuidad: 1) la repetición de la experiencia, para la revisión y la ampliación del glosario, con otros grupos, de forma que se trabaje con el total del alumnado matriculado en la asignatura, sean grupos de mañana o de tarde ${ }^{9}$; 2) la extensión de la práctica docente a otras asignaturas sustantivas del plan docente, dado que la formación de un glosario especializado sobre un listado de términos específicos (de cualquier disciplina o de un aspecto puntual de la misma) es de fácil aplicación a otras asignaturas, y 3) dado que cualquier glosario generado a partir de prácticas como la descrita quedará disponible como recurso académico, mediante un vínculo público para el acceso a través de la red (URL), la posible extensión de la práctica permite la ampliación y la articulación de la «base de conocimientos» que un departamento o facultad puede aportar a las redes mundiales de información.

Los resultados de experiencias posteriores, con utilización de tecnologías wiki, pueden ser consultados en otras publicaciones relacionadas con esta nota de investigación.

8. Que, en un principio, se centraba más en la introducción del uso de nuevas TIC en el aula.

9. Esto permitirá investigar sobre la participación diferencial del alumnado en función de su nivel de asistencia o su perfil tipo (trabajador o estudiante a tiempo completo). 


\section{Referencias bibliográficas}

CABEro, J. (1998). «Las aportaciones de las nuevas tecnologías a las instituciones de formación continuas: Reflexiones para comenzar el debate». En: MARTín-MORENO, Q. et al. (coords.). V Congreso interuniversitario de organización de instituciones educativas. Madrid: Departamentos de Didáctica y Organización Escolar de la Universidad de Alcalá, Complutense.

Harasim, L. Hiltz, S.R. TurofF, M. y Teles, L. «Guía para la enseñanza y el aprendizaje en red", Gedisa, EDIUOC, Barcelona, 2002. (1 a edición en inglés en 1998)

KOZMA, R. y SCHANK, P. (2000). "Conexión con el siglo XXI: la tecnología como soporte de la reforma educativa». En: DedE, Ch. (comp). Aprendiendo con tecnología. Barcelona: Paidós, 25-55.

MARQUÉS, P. (1999). "Criterios para la clasificación y evaluación de espacios web de interés educativo». Educar, 25, 95-111.

PAlloff, R. y PRATT, K. (1999). Building learning communities in cyberspace. San Francisco: Jossey-Bass.

Roblyer, M.; EDWARDS, J. y HARRILNK, M. (1997). Integrating Educational Technology into Teaching. Columbus, Ohio (EEUU): Prentice Hall.

RYAN, S. et al. (2000). The virtual university: The Internet and resource-based learning. Londres: Kogan Page.

SALMON, G. (1998). «Developing learning through effective online moderation». Active Learning, 9, diciembre.

- (2000). E-moderating: The key to teaching and learning online. Londres: Kogan Page.

YÁBAR, J.M. y BARBARÁ, P.L. (1999). La Universidad de la Sociedad de la Información: Retos y respuestas. Actas. II Jornadas de Tecnologías de la Información y Comunicaciones en las Universidades Españolas. Zaragoza: CRUE. 